\title{
ON THE LAMINAR FLOW ANALOGY AND ITS APPLICATION TO LIMIT CYCLE SYNTHESIS*
}

\author{
BY \\ MICHAEL D. GREENBERG \\ Therm Advanced Research, Inc., Ithaca, N. Y.
}

1. Introduction. We consider here the autonomous, nonlinear, second order differential equation

$$
x^{\prime \prime}+\beta\left(x, x^{\prime}\right)=0
$$

the primes denoting differentiation with respect to the time $t$. It has been widely noted in the literature (e.g. [1]) that, by virtue of the presence of a "continuity" relation, the trajectories in the $x, x^{\prime}$ phase plane may be regarded as the streamlines of plane steady flow of a compressible fluid.

In Sec. 2 we will examine the dynamical implications of this interpretation, and it will be shown that an applied force field must be supplied, in general, if the analogy is to be strictly valid.

The kinematical aspects of the flow will then be considered further in Sec. 3, and will suggest a tractable procedure for limit cycle synthesis, i.e. the construction of a function $\beta\left(x, x^{\prime}\right)$ such that the system (1) exhibits a prescribed limit cycle.

2. Laminar flow analogy. Let us regard (1), for the moment, as the equation governing the one-dimensional motion of a unit mass, and introduce the generalized coordinate $q \equiv x$ and generalized momentum $p \equiv \partial L / \partial q^{\prime}=\partial L / \partial x^{\prime}$, where $L\left(x, x^{\prime}, t\right)$ is a Lagrangian of the system. That a Lagrangian does in fact exist may be seen by writing (1) equivalently as $\rho x^{\prime \prime}+\rho \beta=0$, where $\rho\left(x, x^{\prime}, t\right)$ is to be a positive function chosen such that

$$
\begin{aligned}
\rho x^{\prime \prime}+\rho \beta & \equiv \frac{d}{d t}\left(\frac{\partial L}{d x^{\prime}}\right)-\frac{\partial L}{\partial x} \\
& =L_{x^{\prime} x^{\prime}} x^{\prime \prime}+L_{x^{\prime} x} x^{\prime}+L_{x^{\prime} t}-L_{x}
\end{aligned}
$$

with the subscripts denoting partial derivatives. Equating the two $x^{\prime \prime}$ terms, it follows that

$$
L=\iint \rho \partial x^{\prime} \partial x^{\prime}
$$

The quantities $L_{x^{\prime} x}, L_{x^{\prime} t}$ and $L_{x}$ in (2) may therefore be expressed as $\int \rho_{x} \partial x^{\prime}, \int \rho_{t} \partial x^{\prime}$ and $\iint \rho_{x} \partial x^{\prime} \partial x^{\prime}$ respectively. Equating the remaining terms in (2), i.e. the second on the left with the last three on the right, we have

$$
\rho \beta=x^{\prime} \int \rho_{x} \partial x^{\prime}+\int \rho_{t} \partial x^{\prime}-\iint \rho_{x} \partial x^{\prime} \partial x^{\prime}
$$


and, finally, differentiation of (4) with respect to $x^{\prime}$ produces the equation

$$
(\rho \beta)_{x^{\prime}}=x^{\prime} \rho_{x}+\rho_{t}
$$

to be satisfied by $\rho$.

Alternatively, let us start with the well-known result, from classical mechanics, that for a conservative system areas in $q, p$ phase space are conserved; that is,

$$
\iint_{\Omega(t)} d q d p=\text { constant }
$$

where $\Omega(t)$ encloses a given set of representative points as they move about in the $q, p$ plane. Transforming to the $x, x^{\prime}$ phase plane $\Omega(t) \rightarrow \Sigma(t)$ say, and we have

$$
\iint_{\Omega(t)} d q d p=\iint_{\Sigma(t)}\left|L_{x^{\prime} x^{\prime}}\right| d x d x^{\prime}=\text { constant }
$$

since the Jacobian of the transformation is

$$
\frac{\partial(q, p)}{\partial\left(x, x^{\prime}\right)}=\left|\begin{array}{ll}
1 & L_{x^{\prime} x} \\
0 & L_{x^{\prime} x^{\prime}}
\end{array}\right|=L_{x^{\prime} x^{\prime}} .
$$

Now, the second of Eqs. (7) may clearly be interpreted as a continuity condition, with the trajectories in the $x, x^{\prime}$ plane regarded as the streamlines of a plane steady flow of a compressible fluid with mass density $\left|L_{x^{\prime} x^{\prime}}\right|$ and velocity components $u=d x / d t=x^{\prime}$ and $v=d x^{\prime} / d t=-\beta\left(x, x^{\prime}\right)$, respectively. We observe that the mass density $\left|L_{x^{\prime} x^{\prime}}\right|$ is, as a matter of fact, our function $\rho\left(x, x^{\prime}, t\right)$, and that the continuity equation

$$
\rho_{\imath}+\nabla \cdot \rho \mathbf{V}=0
$$

implied by (7), where $\mathbf{V}=(u, v)$, is identical to (5).

In addition to the kinematical condition (9), however, it is also necessary that the dynamical Euler equation

$$
D \mathrm{~V} / D t=-\nabla p / \rho+\mathbf{F}
$$

be satisfied, where $\mathbf{F}\left(x, x^{\prime}\right) \equiv(X, Y)$ is a body force per unit mass, if the laminar flow analogy is to be complete. Taking the fluid to be barotropic, so that $p=f(\rho)$ say, consider for example the $x$-component of the $\nabla p / \rho$ term:

$$
\frac{1}{\rho} \frac{\partial p}{\partial x}=\frac{1}{\rho} \frac{d f}{d \rho} \frac{\partial \rho}{\partial x} \equiv \frac{d g}{d \rho} \frac{\partial \rho}{\partial x}=\frac{\partial}{\partial x} g\left[\rho\left(x, x^{\prime}, t\right)\right] .
$$

Similarly, $p_{x^{\prime}} / \rho$ may be expressed as $g_{x^{\prime}}$ so that the full Euler equations become

$$
\begin{aligned}
& u_{t}+u u_{x}+v u_{x^{\prime}}=-g_{x}+X \\
& v_{t}+u v_{x}+v v_{x^{\prime}}=-g_{x^{\prime}}+Y .
\end{aligned}
$$

The $g$ terms may then be eliminated by taking $\partial / \partial x^{\prime}$ of (12), $\partial / \partial x$ of (13) and subtracting. Recalling that $u=x^{\prime}$ and $v=-\beta$ we finally obtain the following condition on the body force field,

$$
Y_{x}-X_{x^{\prime}}=\beta_{x^{\prime}}-x^{\prime} \beta_{x x}+\beta_{x} \beta_{x^{\prime}}+\beta \beta_{x x^{\prime}} .
$$

It is interesting that $\mathbf{F}$ will be conservative (and, in particular, may be taken equal to 
zero) if and only if the right side of (14) vanishes. Such is the case, for the simple example $\beta=a x^{\prime}+b x$, if $a=0$ or $b=-1$.

3. Application to limit cycle synthesis. Consider a stable limit cycle $\Gamma$ which we will take, for simplicity, to consist of the unit circle $r=\left(x^{2}+x^{\prime 2}\right)^{1 / 2}=1$. Introducing a control volume $\tau$ bounded inside by $r=1-\epsilon$ and outside by $r=1+\epsilon$, we see that there is a steady net mass flux per unit time into $\tau$. Since the continuity equation is to be satisfied throughout the plane, and $\epsilon$ is arbitrarily small, it is clear that the mass density must tend to infinity as we approach $\Gamma$; similarly for noncircular limit cycles, stable or unstable. We emphasize that $\Gamma$ may not be regarded as a distributed "sink", as is often said. On the contrary, we argue that $\rho$ must be infinite on $\Gamma$ because, by virtue of the continuity condition (9), there can be no $\operatorname{sinks}$ in $\tau$ to dispose of the steady influx of fluid. In the original $p, q$ plane, however, such an influx into an annulus would imply the presence of a "sink" since the flow in $p, q$ space is incompressible, as seen from (7). This probably accounts for the confusion.

Thus it appears that one way to determine the precise limit cycle configuration corresponding to a given $\beta\left(x, x^{\prime}\right)$ is to solve (5) for $\rho$, and determine those curves on which $\rho$ is infinite. For the class of $\beta$ 's which are of interest, however, (5) constitutes a difficult partial differential equation.

The synthesis problem, on the other hand, appears attractively simple. The idea is to prescribe a density function $\rho$ with singularities on the desired limit cycles and foci, and to solve (5) for the resulting $\beta$. This time (5) is easily solved, its general solution being

$$
\beta=\rho^{-1}\left\{\int\left(x^{\prime} \rho_{x}+\rho_{t}\right) \partial x^{\prime}+\phi(x)\right\}
$$

with $\phi(x)$ arbitrary.

We can, at least, be assured that the prescribed closed curve will be a trajectory:

Lemma. Let $\Gamma$ be a Jordan curve in the $x, x^{\prime}$ phase plane, and let $N$ be an open set containing $\Gamma$. If $\rho\left(x, x^{\prime}, t\right)$ is prescribed such that $\rho_{x}$ and $\rho_{x^{\prime}}$ are continuous in $N-\Gamma$, $\rho \rightarrow \infty$ for any sequence of points with its limit point on $\Gamma$, and $\beta$ (as given by (15)) is continuous in $N$, then $\Gamma$ will be a trajectory of (1).

Proof. Consider any given point $P$ on $\Gamma$. Since $\beta$ is to be continuous on $\Gamma$, it follows that $\Gamma$ will be smooth at $P$, so that we may define local unit normal and tangent vectors, say $\mathbf{n}$ and $\mathbf{s}$ respectively. These two vectors may, in turn, be used to define a Cartesian coordinate system $n, s$ with its origin at $P$, its $n$-axis aligned with $\mathbf{n}$, and its $s$-axis aligned with s. In terms of this Cartesian system the continuity equation (9) becomes

$$
\rho_{\iota}+\nabla \cdot \rho \mathrm{V}=\rho_{\iota}+\left(\rho V^{s}\right)_{s}+\rho V_{n}^{n}+\rho_{n} V^{n}=0
$$

where $V^{s, n}$ are the $s$ and $n$ fluid velocity components respectively, and the subscripts denote partial derivatives as before. Now, the first three terms of the middle member of (16) are of order $O(\rho)$ in the neighborhood of $P$, so that

$$
V^{n}=O\left(\rho / \rho_{n}\right)=o(1) \text {. }
$$

Since $\beta$ is continuous in $N$ then so must $\mathrm{V}$ and, in particular, $V^{n}$ be continuous there. This, together with (17), implies that $V^{n}=0$ on $\Gamma$. Surely, then, $\Gamma$ is a trajectory of (1).

The conditions of the Lemma are not, however, sufficient to ensure that the tra- 
jectory $\Gamma$ will be a limit cycle. This may be seen from the following example, where we will try to synthesize a limit cycle on $r=1$. It is reasonable to prescribe $\rho=1 / r^{2}\left|r^{2}-1\right|$, with the factor $1 / r^{2}$ included in an attempt to produce an accompanying focus at the origin. From (15), we find $\beta=r^{2}\left|r^{2}-1\right| \phi(x)+x$, which reduces to the simple harmonic oscillator for the choice $\phi=0$, so that the conditions of the Lemma are in fact not sufficient for limit cycle synthesis.

Furthermore, the above $\beta$ does not give the desired limit cycle for any choice of $\phi(x)$. This is seen from the appropriate form of Poincare's well-known condition for asymptotic orbital stability of a periodic solution,

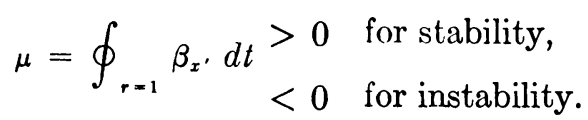

In our case $\beta_{x^{\prime}}$ is proportional to $x^{\prime} \phi(x)$ on $r=1$ so that the integral vanishes by symmetry, precluding the possibility of a limit cycle on $r=1$.

It is interesting to interpret the Poincaré condition (18) in terms of our flow analogy. Notice that

$$
\frac{D \rho}{D t}=\frac{\partial \rho}{\partial t}+\mathrm{V} \cdot \nabla \rho=\rho_{t}+x^{\prime} \rho_{x}-\beta \rho_{x^{\prime}}=\rho \beta_{x^{\prime}}
$$

where the last equality follows from (5). Integrating, it follows that

$$
\rho=\rho_{0} \exp \int_{t_{0}}^{t} \beta_{x^{\prime}} d t \text {. }
$$

In the above example, where $\rho=1 / r^{2}\left|r^{2}-1\right|$ and $\beta=x$ say, we have $\beta_{x^{\prime}}=0$ so that $\rho=$ constant for each fluid particle. Thus the unboundedness of $\rho$ on $\Gamma$ was to no avail since we did not also have $D \rho / D t>0$ (for a stable limit cycle, for example) over "most" of the neighborhood of $\Gamma$, in which case the density of each particle would increase indefinitely and the particle would be obliged to asymptotically approach $\Gamma$. The Poincaré condition supplies this additional requirement.

Returning to the problem of synthesizing a limit cycle on the unit circle, let us consider the more general class of densities which are of the form $\rho=\rho(r)$ and which satisfy the conditions of the Lemma. From (15) we find that $\beta=\rho^{-1}(r) \phi(x)+x$ so that

$$
\mu=\left.\frac{d}{d r} \rho^{-1}\right|_{r-1} \oint_{r=1} x^{\prime} \phi(x) d t .
$$

Now, if $\rho=O(r-1)^{-\alpha}$ near $r=1$, then the $(d / d r) \rho^{-1}$ factor will be finite for $\alpha \geq 1$ and infinite for $\alpha<1$. In either case the integral is identically zero. Thus $\mu=0$ for $\alpha \geq 1$ and no limit cycle is obtained, whereas for $\alpha<1$ the right hand side of (21) is indeterminate. This indeterminateness is symptomatic of the fact that the Poincare condition does not apply for $\alpha<1$. Specifically, the proof of the Poincare condition breaks down at the outset because a "first approximation" to $\beta$ does not exist. In this case we merely conjecture that $\mu^{-}>0(<0)$ implies stability (instability) from the inside, and that $\mu^{+}>0(<0)$ implies stability (instability) from the outside, where $\mu^{ \pm}$are defined by (18) with $r=1$ replaced by $r=1^{ \pm}$respectively. This would indicate that no limit cycle results, again, for $\alpha<1$.

Instead of pursuing this point further, let us consider a more promising form for $\rho$. Notice that wo have allowed for an explicit time dependence in $\rho\left(x, x^{\prime}, t\right)$ despite the fact that $\beta$ is to be autonomous. We observe, from (15), that $\rho\left(x, x^{\prime}, t\right)$ will, in fact, 
lead to an autonomous $\beta$ if it is of the form $f\left(x, x^{\prime}\right) \exp \lambda t$ and if we choose $\phi(x)=0$. Consider, for example,

$$
\rho=A(r) \exp \lambda t=\left|r^{2}-1\right|^{-1 / 2} \exp \lambda t
$$

with $\lambda$ a constant which we will permit to take different values inside and outside the unit circle. From (15) then, with $\phi=0, \beta$ may be expressed as

For $r \leq 1$,

$$
\beta=\left|r^{2}-1\right|^{1 / 2} g\left(x, x^{\prime}\right)+x .
$$

$$
g\left(x, x^{\prime}\right) \equiv \lambda_{i} \sin ^{-1} x^{\prime}\left(1-x^{2}\right)^{-1 / 2}
$$

which contains a critical point only at the origin. Since " $\alpha$ " $=\frac{1}{2}$ is $<1$ we will avoid the Poincaré condition (as well as its conjectured extension) in verifying that $r=1$ is a limit cycle and in ascertaining its stability. Near the origin, $\beta \sim \lambda_{i} x^{\prime}+x$. For $\lambda_{i}<0$ the origin is therefore an unstable focus and the cycle $r=1$ is stable from the inside; conversely for $\lambda_{i}>0$. Alternatively, we note that

$$
\begin{aligned}
D\left(r^{2}\right) / D t & =2 x^{\prime}(x-\beta) \\
& =-2 \lambda_{i} x^{\prime}\left(1-r^{2}\right)^{1 / 2} \sin ^{-1} x^{\prime}\left(1-x^{2}\right)^{-1 / 2}
\end{aligned}
$$

which is positive (negative) throughout $r<1$ for $\lambda_{i}<0(>0)$. Notice that the focus was obtained without including an explicit singularity in $\rho$ at the origin; it is accounted for implicitly by the exp $\lambda t$ factor which $\rightarrow \infty$ as $t \rightarrow+\infty(-\infty)$ for $\lambda>0(<0)$.

Unfortunately, $g\left(x, x^{\prime}\right)$ contains logarithmic singularities, for $r>1$, along the two lines defined by $|x|=1, x^{\prime}<0$. The corresponding behavior of $\beta$ may be regarded as objectionable. It may, however, be removed by choosing an $A(r)$ which is not so strongly singular on $r=1$.

In particular, let us choose

$$
A(r)=\log \left|\frac{r^{2}+2}{r^{2}-1}\right|
$$

shown below in Fig. 1. This time we obtain

$$
\beta=h\left(x, x^{\prime}\right) / \log \left|\frac{r^{2}+2}{r^{2}-1}\right|+x
$$

where

$$
\begin{aligned}
h\left(x, x^{\prime}\right) \equiv \lambda\left\{x^{\prime} \log \left|\frac{r^{2}+2}{r^{2}-1}\right|+2\left(x^{2}+2\right)^{1 / 2} \tan ^{-1} \frac{x^{\prime}}{\left(x^{2}+2\right)^{1 / 2}}+\Lambda\right\} \\
\Lambda\left(x, x^{\prime}\right) \equiv\left(1-x^{2}\right)^{1 / 2} \log \left|\frac{x^{\prime}-\left(1-x^{2}\right)^{1 / 2}}{x^{\prime}+\left(1-x^{2}\right)^{1 / 2}}\right|, \quad|x| \leq 1 \\
\equiv-2\left(x^{2}-1\right)^{1 / 2} \tan ^{-1} \frac{x^{\prime}}{\left(x^{2}-1\right)^{1 / 2}}, \quad|x|>1
\end{aligned}
$$

and $\lambda=\lambda_{i}, \lambda_{0}$ inside and outside the unit circle, respectively. The only critical point occurs at the origin, where $\beta \sim \lambda_{i} x^{\prime}+x$ again. Thus the cycle $r=1$ is stable from the inside for $\lambda_{i}<0$ and unstable for $\lambda_{i}>0$. Furthermore, it is stable from the outside for $\lambda_{0}>0$ and unstable for $\lambda_{0}<0$, as can be seen from the form of $D\left(r^{2}\right) / D t$. Despite the disappointingly cumbersome form of $\beta$, we observe that it does exhibit the desired limit cycle and is, at the same time, a continuous function of $x$ and $x^{\prime}$. 


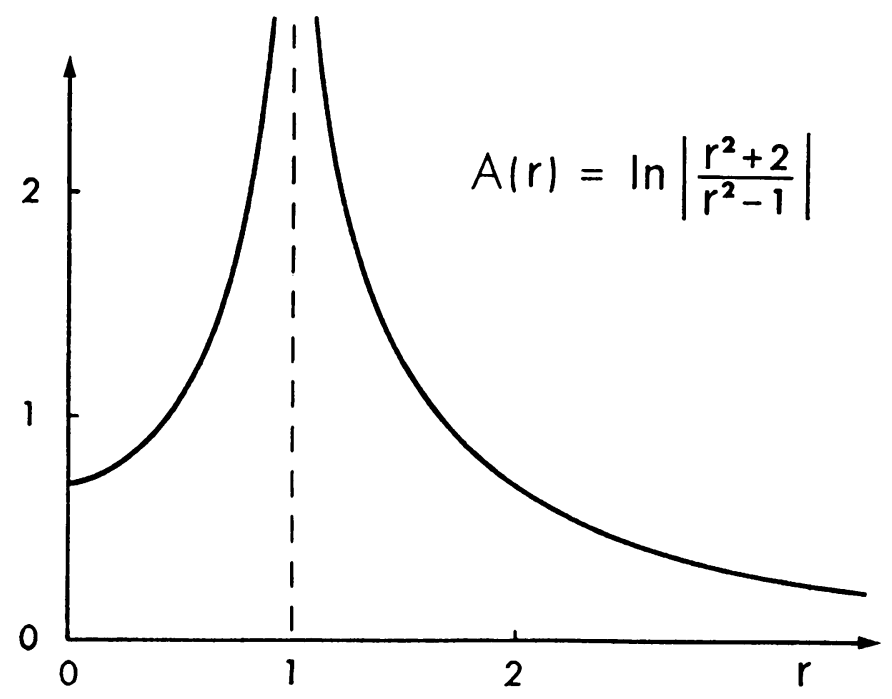

FIg. 1. Density Function Factor.

4. Comparison with Kaplan's work. The above adaptation of the flow analogy bears a resemblance to certain earlier work by Kaplan [2]. He considers the system

$$
x_{1}^{\prime}=f_{1}\left(x_{1}, x_{2}\right), \quad x_{2}^{\prime}=f_{2}\left(x_{1}, x_{2}\right)
$$

(actually an $n$th order system, but we take $n=2$ for the sake of comparison) and bases his approach upon the existence of an integral of (28); i.e. a function $F\left(x_{1}, x_{2}, t\right)$ which remains constant along each solution curve. Roughly, his idea is that a given initial distribution $F\left(x_{1}, x_{2}, 0\right)$ will get "smeared" onto a stable limit cycle $\Gamma$ as $t \rightarrow \infty$, so that $F \rightarrow 0$ except on $\Gamma$. If $F\left(x_{1}, x_{2}, t\right)$ can be determined, this permits the determination of the limit cycle $\Gamma$. The function $F$ may be computed as the solution of the partial differential equation

$$
\frac{D F}{D t}=\frac{\partial F}{\partial t}+\mathrm{V} \cdot \nabla F=0
$$

subject to the given initial condition, where $\mathbf{V}=\left(f_{1}, f_{2}\right)$. If we set $f_{1}=x_{2}=x^{\prime}$ and $f_{2}=-\beta$ this may be expressed as

$$
(F \beta)_{x^{\prime}}=x^{\prime} F_{x}+F_{t}+F \beta_{x^{\prime}} \text {. }
$$

Comparing with (5), we observe that the "density function" $F$ may be interpreted as the fluid mass density only if $\beta_{x^{\prime}}=0$, which precludes the possibility of limit cycles and is therefore of no interest here.

Although the physical interpretation of Kaplan's work differs slightly from that of the present work, the two approaches are nevertheless quite similar.

5. Acknowledgement. The author is greatly indebted to Professors H. D. Block and T. P. Mitchell for suggesting the basic framework of the present analysis.

\section{REFERENCES}

1. A. Andronow, and C. Chaikin, Theory of oscillations, Princeton Univ. Press, Princeton, 1949, pp. 86-89

2. W. Kaplan, Some methods for the analysis of the flow in phase space, Proc. Symp. on Nonlinear Circuit Analysis, Polytechnic Institute of Brooklyn, Brooklyn, 1953, pp. 99-106 